\title{
Myofibroblast contraction is essential for generating and regenerating the gas-exchange surface
}

\author{
Rongbo Li, ${ }^{1}$ Xiaoping Li, ${ }^{1}$ James Hagood, ${ }^{1,2}$ Min-Sheng Zhu, ${ }^{3,4,5}$ and Xin Sun ${ }^{1,6}$ \\ 'Department of Pediatrics, School of Medicine, UCSD, La Jolla, California, USA. 2Division of Pulmonology, Department of Pediatrics, University of North Carolina-Chapel Hill, Chapel Hill, North Carolina, \\ USA. ${ }^{3}$ State Key Laboratory of Pharmaceutical Biotechnology, ${ }^{4}$ Model Animal Research Center, and ${ }^{5} \mathrm{MOE}$ Key Laboratory of Model Animal for Disease Study, Nanjing University, Nanjing, China. ${ }^{6}$ Division of \\ Biological Sciences, UCSD, La Jolla, California, USA.
}

\begin{abstract}
A majority ( $95 \%)$ of the gas-exchange surface area is generated through septa formation during alveologenesis. Disruption of this process leads to alveolar simplification and bronchopulmonary dysplasia (BPD), a prevalent disorder in premature infants. Although several models have been proposed, the mechanism of septa formation remains under debate. Here we show that inactivation of myosin light chain kinase (MLCK), a key factor required for myofibroblast contraction, disrupted septa formation, supporting the myofibroblast contraction model of alveologenesis. The alveoli simplification phenotype was accompanied by decreased yes-associated protein (YAP), a key effector in the Hippo mechanotransduction pathway. Expression of activated YAP in Mlck-mutant lungs led to partial reversal of alveolar simplification. In the adult, although Mlck inactivation did not lead to simplification, it prevented reseptation during compensatory regrowth in the pneumonectomy model. These findings revealed that myofibroblast reactivation and contraction are requisite steps toward regenerating the gas-exchange surface in diseases such as BPD and chronic obstructive pulmonary disease (COPD).
\end{abstract}

\section{Introduction}

An average human lung is composed of approximately $143 \mathrm{~m}^{2}$ $\left(\sim 1500 \mathrm{ft}^{2}\right)$ of gas-exchange surface area (1). Approximately $95 \%$ of this surface is generated during the last step of lung development termed alveologenesis, in which millions of septa subdivide the walls of gas-exchange units to form about 480 million alveoli. In premature infants, the process of alveologenesis is either truncated or eliminated, disrupting septa formation, which leads to poor gas exchange. Each year in the United States, an estimated 10,000 to 15,000 premature infants with severe abnormalities of gas exchange are diagnosed with bronchopulmonary dysplasia (BPD), a condition that requires prolonged ventilatory support and is often linked with long-term morbidities such as increased susceptibility to respiratory infections (2).

The process of alveologenesis has been extensively studied using mice, in which alveologenesis initiates on P3 and continues until P39 $(3,4)$. The alveolar surface is commonly depicted in $2 \mathrm{D}$ sections, where secondary septa appear as fingers that protrude into the lumen. The tip of the "finger" is often positive for $\alpha$-smooth muscle actin ( $\alpha$-SMA), a product of the actin $\alpha 2$ (Acta2) gene and a marker for secondary crest myofibroblasts. A reduction in myofibroblasts, as was detected in PDGF $\alpha$ - and PDGF receptor $\alpha$-mutant ( $P d g f a$ - and $P d g f r a$-mutant) lungs, or our genetic ablation of myofibroblasts through expression of diphtheria toxin in these cells, disrupted septa formation (5-9). These data demonstrate that myofibroblasts are essential for secondary septa formation.

Conflict of interest: The authors have declared that no conflict of interest exists. Copyright: () 2020, American Society for Clinical Investigation.

Submitted: July 31, 2019; Accepted: February 13, 2020; Published: April 27, 2020

Reference information: / Clin Invest. 2020;130(6):2859-2871.

https://doi.org/10.1172/JCl132189.
Several models that were largely based on a 2D presentation of secondary septa as fingers were proposed to depict how these fingers form. One model proposed that they arise through cellular repulsion from the alveolar wall into the lumen (10). A second model proposed that they arise through sprouting and growth of the tips (11). A third model proposed that these fingers arise through ballooning of the alveolar wall, leading to septa elongation at the basal end, away from fixed luminal tips (12). In contrast, our recent 3D imaging analysis depicted secondary septa as ridges that underlay thinly stretched, long myofibroblasts (13). These myofibroblasts join together in a pattern of interconnected rings. This 3D pattern resembles a "fishnet," which was first articulated in 1970 by Emery on the basis of an observation of histological sections of human alveoli (14). We renewed this model based on 3D reconstruction of confocal images and propose that myofibroblasts constitute the "strings" of the fishnet. In this model, the network is elaborated as new myofibroblasts are added. More important, coordinated contraction of the myofibroblasts either tightens the net and/or holds it in place against air pressure on alveolar walls, leading to the rise of septal ridges that are directly over the strings relative to areas that are in between strings (13).

To directly test this revised model and identify the fundamental mechanism of septa formation, we focused on myofibroblast contraction, a hallmark characteristic of differentiated myofibroblasts. A key prediction for this model postulates that preventing myofibroblast contraction should lead to a failure in septa formation. In many other tissue settings, myofibroblast contraction is regulated by a network of signaling pathways directed to control the molecular motor myosin (15). Phosphorylation of myosin light chain (MLC) activates actomyosin Mg-ATPase, resulting in the sliding of actin-myosin filaments and force generation. The extent of MLC phosphorylation, which is regulated by the counteracting activity of myosin light 
A PdgfrartTA;tetO-cre;Rosa ${ }^{m T m G}$

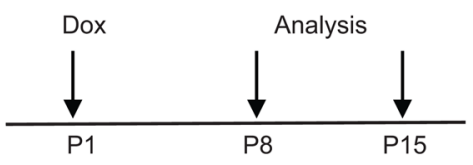

H

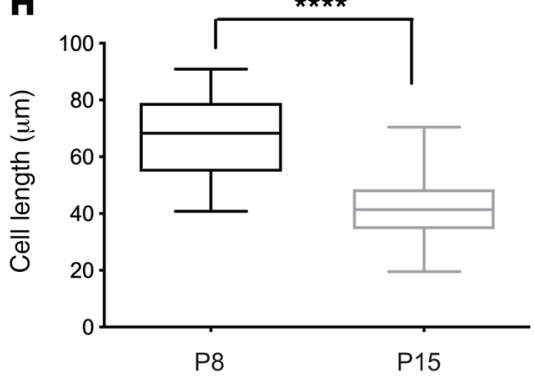

I Tbx4-rtTA;tetO-cre;Mlck $k^{\text {th }}$
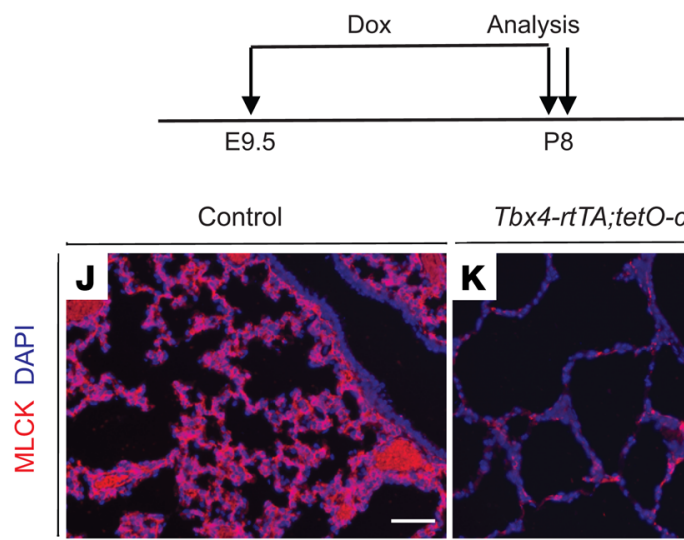

Tbx4-rtTA;tetO-cre;Mlck ${ }^{\text {t/f }}$
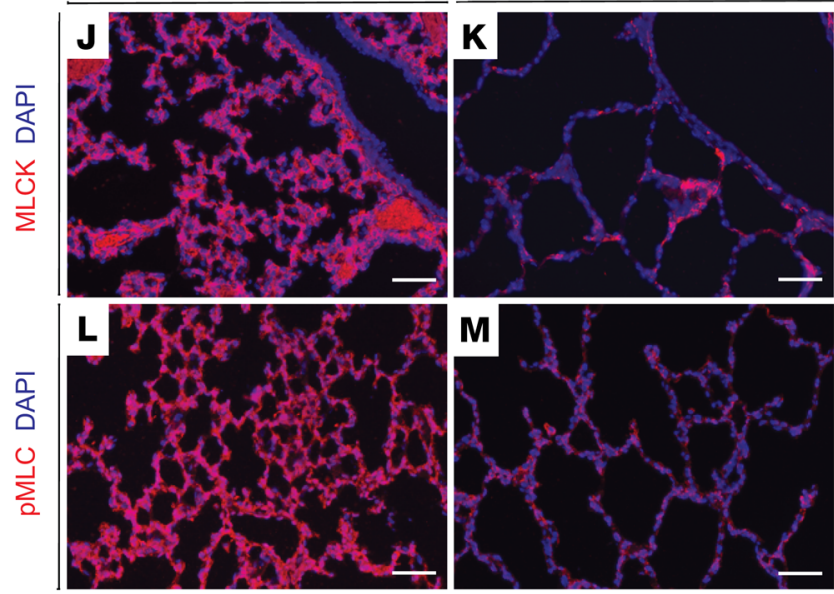

$\mathbf{N}$
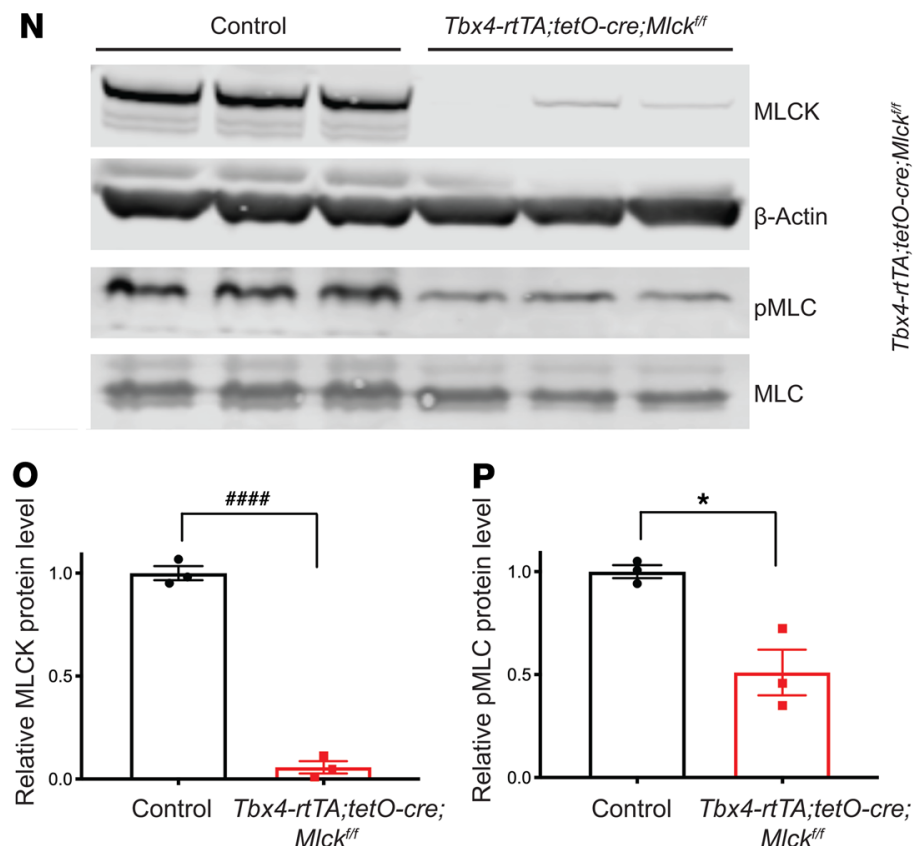
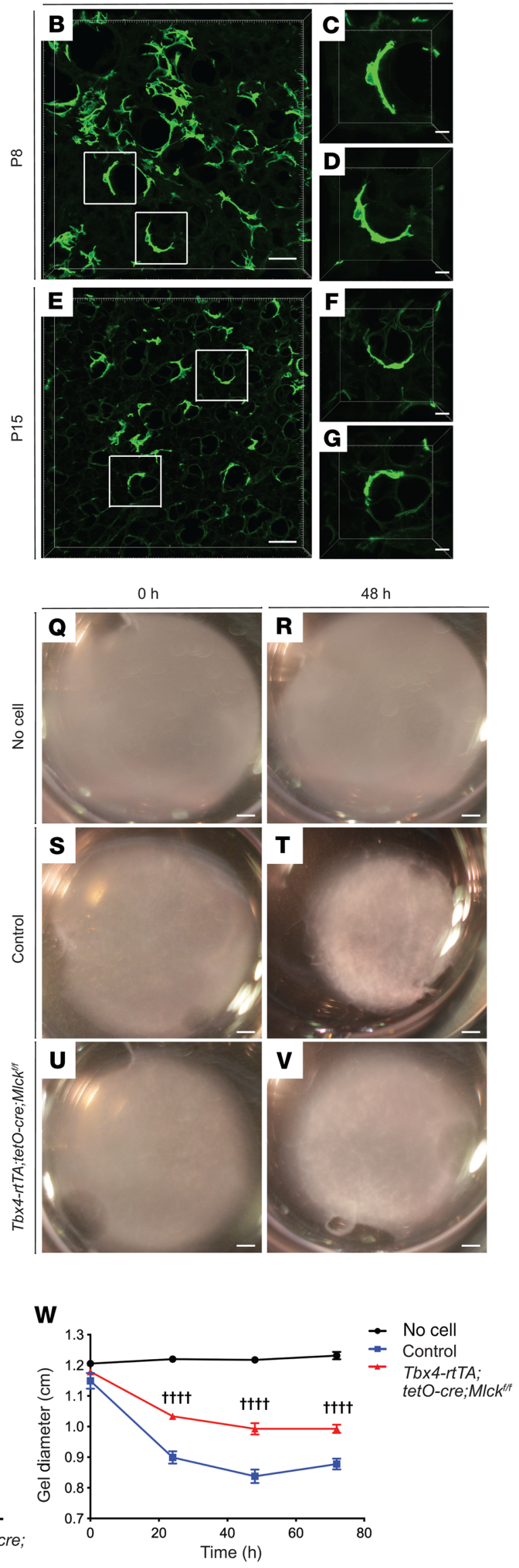
Figure 1. Mlck inactivation leads to decreased myofibroblast contraction. (A) Timeline of dox treatment and analysis of PdgfrattA tetO-Cre Rosa ${ }^{m T m C}$ pups for assessment of cell morphology. (B-C) Representative images of reconstructed $70-\mu \mathrm{m} z$-stacks of GFP pattern (green) in the alveolar region of lungs at P8 (B-D) and P15 (E-G). (H) Quantification for images in B-C. ${ }^{* * *} P=0.0000005(n=20$ cells per stage selected from 3 samples at $P 8$ and 2 samples at P15). (I) Timeline of dox-mediated gene inactivation and analysis. (J-M) Representative immunofluorescence staining for MLCK ( $\mathbf{J}$ and $\mathbf{K}$ ) and p-MLC ( $\mathbf{L}$ and $\mathbf{M}$ ) in the alveolar region of control and mutant lungs at $\mathrm{P} 8$. (N) Western blot analysis of lung tissues using antibodies against MLCK, $\beta$-actin, p-MLC, and MLC. (0) Western blot quantification indicated that MLCK levels were decreased in mutant

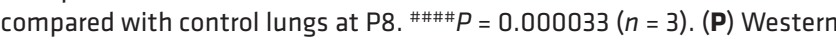
blot quantification indicated a decrease in the ratio of $p-M L C$ to MLC levels in mutant compared with control lungs at $P 8 .{ }^{*} P=0.0133(n=3)$. (Q-V) Representative collagen gel images for no cells, control PdgfraCFP positive myofibroblasts and mutant $P d g f r{ }^{C F P}$-positive myofibroblasts at $\mathbf{O}$ hours and 48 hours. (W) Quantification for images in $\mathbf{Q}-\mathbf{V}$. ${ }^{+t+t} P<$ 0.0001 , control versus Tbx4-rtTA tetO-Cre Mlck ${ }^{f l / f l}$ at 24 hours $(n=3)$; ${ }^{t+t t} P<0.0001$, control versus Tbx4-rtTA tetO-Cre Mlck ${ }^{f l / f l}$ at 48 hours $(n=3) ;{ }^{\dagger+t \dagger} P<0.0001$, control versus Tbx4-rtTA tetO-Cre Mlck fl/fl at 72 hours $(n=3)$. Scale bars: $50 \mu \mathrm{m}(\mathbf{B}, \mathbf{E}$, and J-M), $10 \mu \mathrm{m}$ (C, D, F, and G), and $1 \mathrm{~mm}(\mathbf{Q}-\mathbf{V})$. Data represent the mean \pm SEM. $P$ values were determined by 2-tailed Student's $t$ test $(\mathbf{H}, \mathbf{O}$, and $\mathbf{P}$ ) and 2-way ANOVA with Tukey's method to adjust for multiple comparisons (W).

chain kinase (MLCK) and myosin light chain phosphatase (MLCP), is thought to be the primary determinant for the production of contraction. In support of this, smooth muscle-specific inactivation of the Mlck gene (also termed Mylk) resulted in impaired gut and airway smooth muscle contraction $(16,17)$.

In this study, we show that inactivation of Mlck in the lung mesenchyme disrupted myofibroblast contraction. This led to a drastic halt of septa formation. Furthermore, we found that yes-associated protein (YAP), the core transcription factor in the Hippo pathway, is regulated by MLCK and in turn functions in parallel with MLCK to control alveologenesis. Last, in a pneumonectomy (PNX) model of compensatory alveolar regrowth, inactivation of Mlck also disrupted reseptation, suggesting the possibility that stimulation of reappearance and coordinated contraction of secondary crest myofibroblasts in the adult may be a strategy to drive new septa formation.

\section{Results}

Dynamic myofibroblast morphology during alveologenesis. To study the role of secondary crest myofibroblasts during alveologenesis, we first analyzed their shape and size. Previously, we generated the mouse line featuring knockin of PdgfrartTA which is active in myofibroblasts during alveologenesis (7). Using this mouse line in combination with tetO-Cre and the $\operatorname{Ros}^{m T m G}$ reporter, we outlined the membranes of myofibroblasts with myristoylated GFP (ref. 18 and Figure 1, A-G). Low-dose doxycycline (dox) injection was given on P1 for sparse labeling of cells, and the lungs were analyzed on P8 and P15. Myofibroblast lengths were significantly shorter on P15 compared with lengths on P8 (Figure 1H), consistent with contraction of these cells.

Inactivation of Mlck reduced myofibroblast contraction. To identify the mechanism underlying myofibroblast contraction, we inactivated Mlck, a key node in smooth muscle cell contraction. This was carried out specifically in the lung mesenchyme using the Tbx4-
rtTA tetO-Cre driver (19). To ensure robust inactivation, dox food was administered starting on E9.5, shortly after lung bud initiation, until P8, the peak of alveologenesis and time of analysis (Figure 1I). We used an anti-MLCK antibody and found that MLCK staining was substantially diminished in Mlck-mutant lungs (Figure 1, J and $\mathrm{K})$. This was corroborated by Western immunoblot analysis, which showed that MLCK protein levels were significantly reduced in the mutant (Figure 1, N and O). Phosphorylated MLC (p-MLC), a primary downstream target of MLCK activity, was also decreased in the mutant lung (Figure 1, L-N and P). To determine whether inactivation of Mlck resulted in impaired myofibroblast contraction, we FACS purified $\mathrm{GFP}^{+}$myofibroblasts from $\mathrm{P} 7 \mathrm{Pdgfra}{ }^{\mathrm{GFP} /+}$ lungs of control mice or mice on a Mlck-mutant background (20). These cells were embedded in collagen gels and cultured for 72 hours and the resulting gel size measured. Gels containing Mlck-mutant myofibroblasts showed statistically significant attenuation of contraction compared with control cells (Figure 1, Q-W). For in vivo validation, we inactivated Mlck using the Pdgfra ${ }^{r t T A}$ tetO-Cre driver with the RosamTmG reporter. Myofibroblast lengths were significantly longer in Mlck-mutant lungs than those in control lungs on P8 (Supplemental Figure 1, A-G; supplemental material available online with this article; https://doi.org/10.1172/JCI132189DS1). Together, these data suggest that $M l c k$ is required for alveolar myofibroblast contraction.

Inactivation of Mlck disrupted septa formation. To determine the role of Mlck at the tissue level during lung development, we inactivated Mlck by administering dox starting on E9.5. By E13.5, Mlck lungs had a normal branching pattern (Supplemental Figure $1 \mathrm{H})$, despite the efficient MLCK inactivation shown by antibody staining (Supplemental Figure 1, I and J), suggesting that Mlck is not required for branching morphogenesis. Mlck pups were viable, and mutant lungs had alveolar simplification starting on P3, a phenotype that was more pronounced by P8 (Figure 2, A-L). Quantification by mean linear intercept (MLI) analysis indicated that the MLI of Mlck-mutant lungs was not significantly different from that of the controls on P1 (Figure 2C). But by P3, P4, and P8, mutant lungs showed a $27 \%, 57 \%$, and $133 \%$ increase, respectively, in relative MLI compared with control lungs (Figure 2, F, I, and L). To complement this genetic approach, we treated WT pups with ML-7, an inhibitor of MLCK, from P1 to P7. ML-7treated lungs showed alveolar simplification by P8 compared with vehicle-treated lungs (Figure 2, M-O), consistent with findings from the $M l c k$-mutant lungs. To determine cellular changes that accompanied alveolar simplification in Mlck-mutant lungs, we assayed for the pattern of cell type-specific markers by a genetically encoded fluorescence marker or immunofluorescence staining. By P8, the myofibroblast marker PDGFR $\alpha$-GFP, the pericyte marker PDGFR $\beta$, the alveolar type 1 epithelial cell marker PDPN, the alveolar type 2 epithelial cell marker SPC, the macrophage marker IsoB4, the endothelial cell marker ICAM2, and the lipofibroblast marker ADRP all appeared normal in proportion to simplification in mutant lungs as compared with control lungs (Supplemental Figure 1, K-V).

To determine the status of myofibroblast cells, we tested for the expression of $\alpha$-SMA, a myofibroblast marker. In 2D sections of control lungs, we detected densely populated $\alpha$-SMA signal, primarily at the tips of secondary septa. However, in Mlck-mutant 

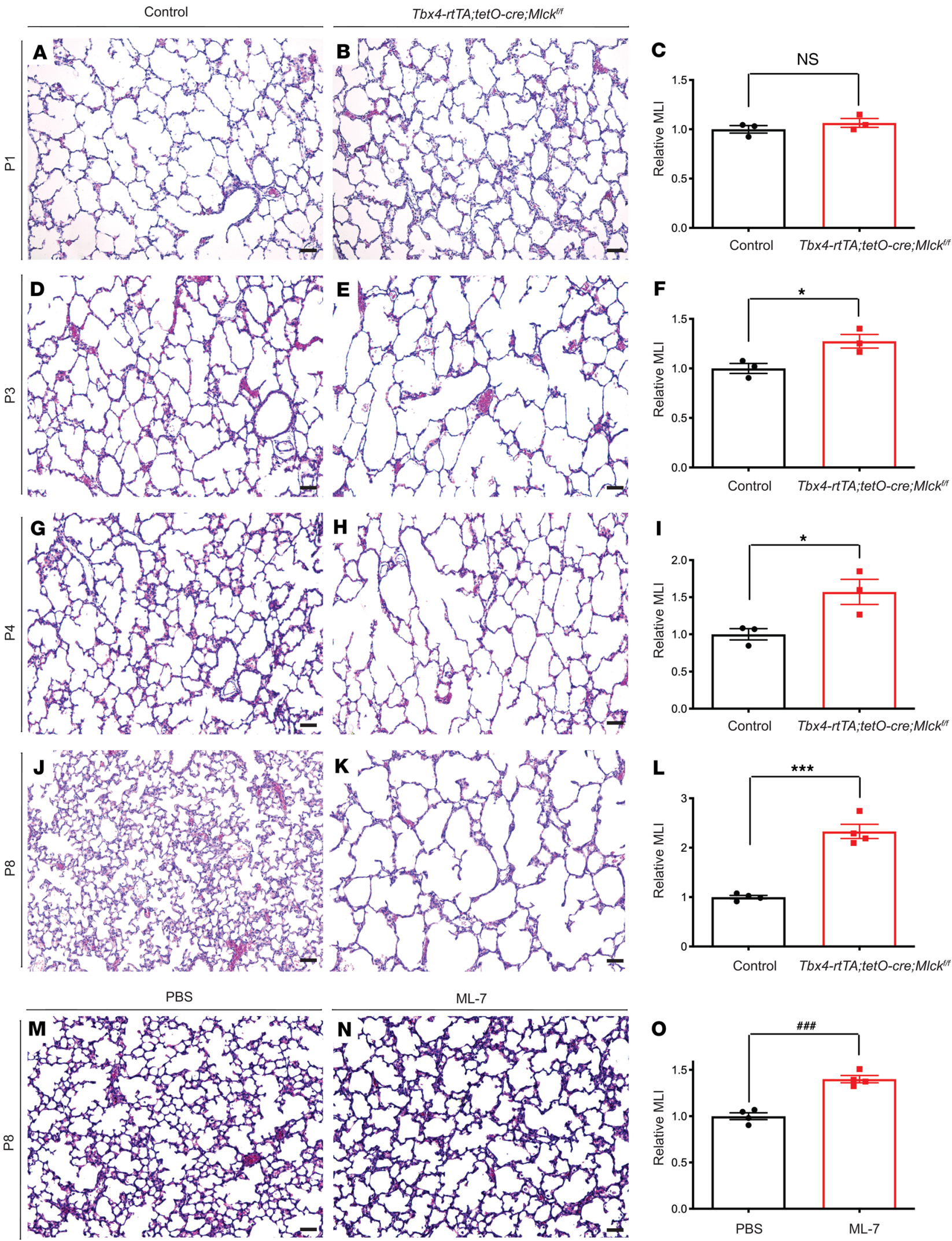

Figure 2. Inactivation of Mlck disrupts alveologenesis. (A-K) Representative H\&E-stained sections from the alveolar regions of control (A, D, G, and J) and mutant (B, E, $\mathbf{H}$, and $\mathbf{K})$ mouse lungs on P1, P3, P4, and P8. (C, F, I, and $\mathbf{L})$ Quantification of alveolar simplification by $\mathrm{MLI}$ on P1 $(P=0.3336[N S], n=3)$, P3 $\left({ }^{*} P=0.0326, n=3\right)$, P4 $\left({ }^{*} P=0.0368, n=3\right)$, and P8 $\left({ }^{* *} P=0.0001, n=4\right)$. (M and $\left.\mathbf{N}\right)$ Representative H\&E-stained sections from the alveolar regions of PBS control- and ML-7-treated mouse lungs on P8. (0) Quantification of alveolar simplification by MLI (\#\# $P=0.0003, n=4)$. Data represent the mean \pm SEM. $P$ values were determined by 2-tailed Student's $t$ test. Scale bars: $50 \mu \mathrm{m}$. 

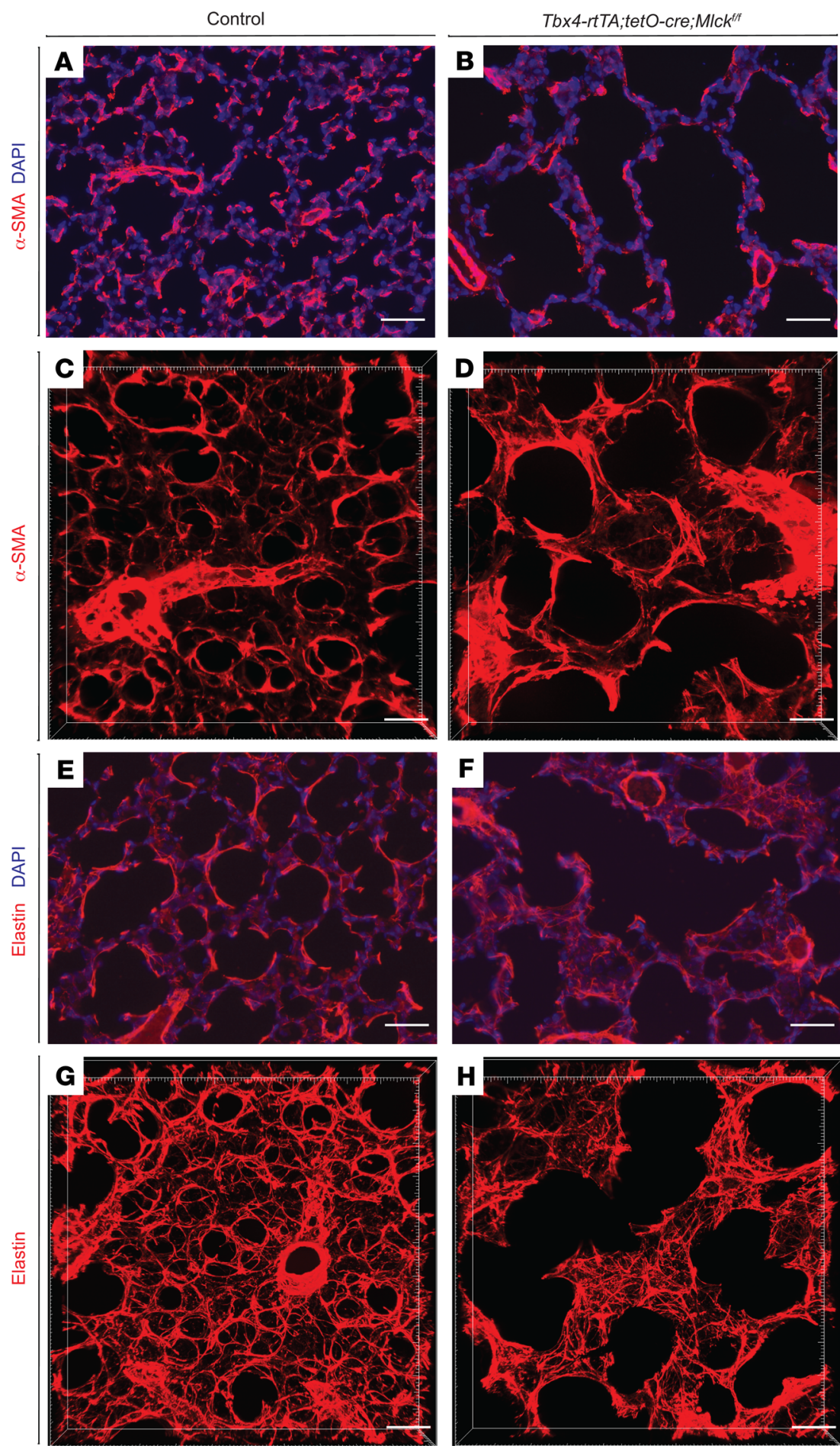

Figure 3. Inactivation of MIck leads to abnormal myofibroblast and elastin patterns. ( $A$ and $B$ )

Representative immunofluorescence staining for $\alpha-S M A$ (red) in sections of the alveolar region showing localization of myofibroblasts on P8. (C and D) Reconstructed $70-\mu \mathrm{m} z$-stacks of immunofluorescence staining for $\alpha$-SMA (red) in the alveolar region of lungs on P8. $\alpha$-SMA staining in the Mlck-mutant lung was less tightly organized compared with control lung. (E and F) Representative immunofluorescence staining for elastin (red) in sections of the alveolar region on P8. (G and $\mathbf{H}$ ) Reconstructed 70- $\mu \mathrm{m}$ z-stacks of immunofluorescence staining for elastin (red) in the alveolar region of lungs on P8. Elastin staining in the Mlck-mutant lung was disorganized compared with control lung. Scale bars: $50 \mu \mathrm{m}$.

extracellular matrix molecule in alveologenesis, as observed in the 2D section and in a more pronounced manner in the 3D reconstruction (Figure $3, \mathrm{E}-\mathrm{H})$. In contrast to tight fibers that underlay alveolar entrance rings in the control, in the mutant, elastin fibers were frayed and extended to other regions of the alveolar walls.

Inactivation of Mlck in phase 1 but not phase 2 of alveologenesis led to alveolar simplification. Our previous results suggested that myofibroblast behavior may be distinct in the 2 phases of alveologenesis (13). In the first phase (P3-P14), myofibroblasts express $\alpha$-SMA fibers, and the elastin network is remodeled from dispersed thin fibers into thick fibers that surround alveolar entrance rings (13). In the second phase (P15-P39), there is no alveolar $\alpha$-SMA signal, whereas the elastin fiber matrix continues to increase in complexity through addition of smaller intervening fibers that underlie new septal ridges. To determine when Mlck is required, we inactivated Mlck at different time points. First, a single dose of dox injection was administered on P1, before the start of alveologenesis, and the lungs were analyzed on P8 (Figure 4A). These Mlck-mutant mice exhibited simplified alveoli (Figure 4, B, $\mathrm{C}$, and $\mathrm{H}$ ), abnormal $\alpha$-SMA patterns (Figure 4 , $\mathrm{D}$ and $\mathrm{E}$ ), and disorganized elastin fibers (Figure $4, F$ and $G$ ), recapitulating the phenotypes in mutants with embryonic inactivation of the gene. Second, 3 daily dox injections were given from P15 to P17, and the lungs were analyzed

lungs, $\alpha$-SMA signal was sparse, possibly as a result of simplification (Figure 3, A and B). This difference in pattern is more comprehensively depicted in $3 \mathrm{D}$ (13). In control lungs on P8, we found that $\alpha$-SMA signal was in tightly packed rings at the alveolar entrance. Many of these rings were interconnected with each other to give rise to a "fishnet" under the alveolar surface (Figure 3C). However, in Mlck-mutant lungs, thicker and more disorganized $\alpha$-SMA cables underlay not just the alveolar entrance ring, but also other regions of the alveolar wall (Figure 3D). These changes in $\alpha$-SMA pattern were accompanied by an altered elastin pattern, the key on P40 (Figure 4I). These late Mlck-mutant lungs were histologically indistinguishable from control lungs (Figure 4, J, K, and N), even though anti-MLCK staining showed a clear decrease in Mlck expression in the mutant lungs, indicative of efficient inactivation (Figure 4, $\mathrm{L}$ and $\mathrm{M}$ ). These results showed that $M l c k$ is required in phase 1 but not phase 2 of alveologenesis.

Inactivation of Mlck led to decreased TGF- $\beta$ signaling. We next sought to determine whether any signaling pathways are disrupted that may mediate alveolar simplification in Mlck-mutant lungs. TGF- $\beta$ signaling has been linked to myofibroblast contraction 
A

Tbx4-rtTA;tetO-cre;Mlck fff

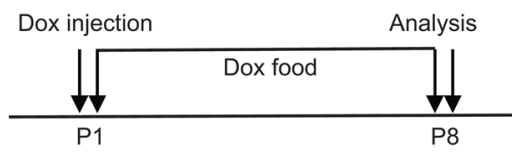

H

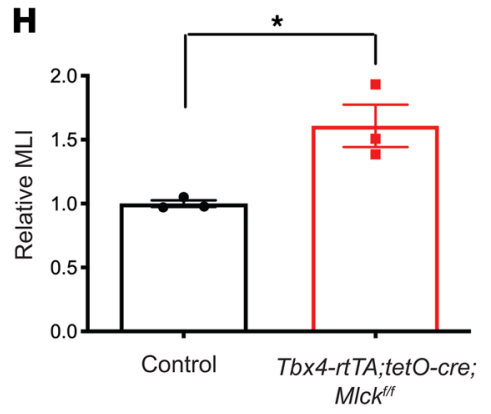

I

Tbx4-rtTA;tetO-cre;Mlck ${ }^{\prime \prime \prime}$
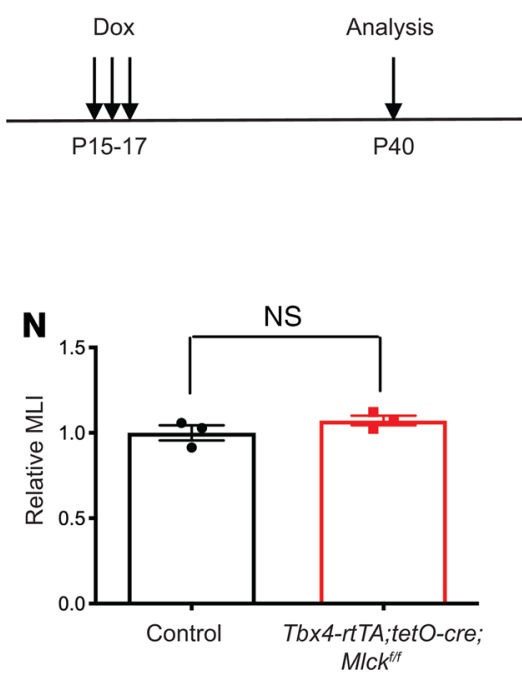

Control

Tbx4-rtTA;tetO-cre;Mlckit

$\underset{\text { Ш }}{\stackrel{\varpi}{I}}$
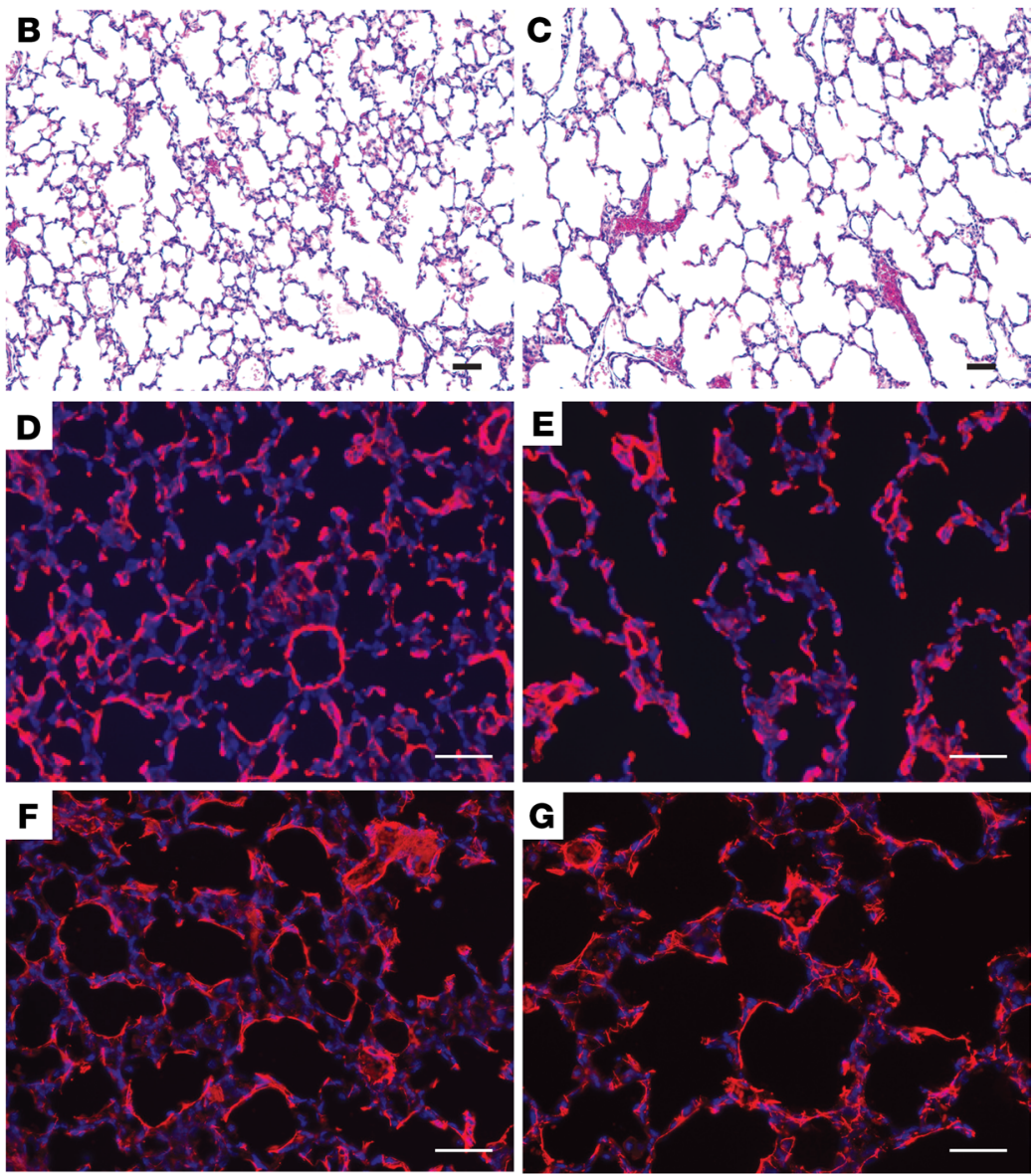

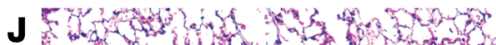
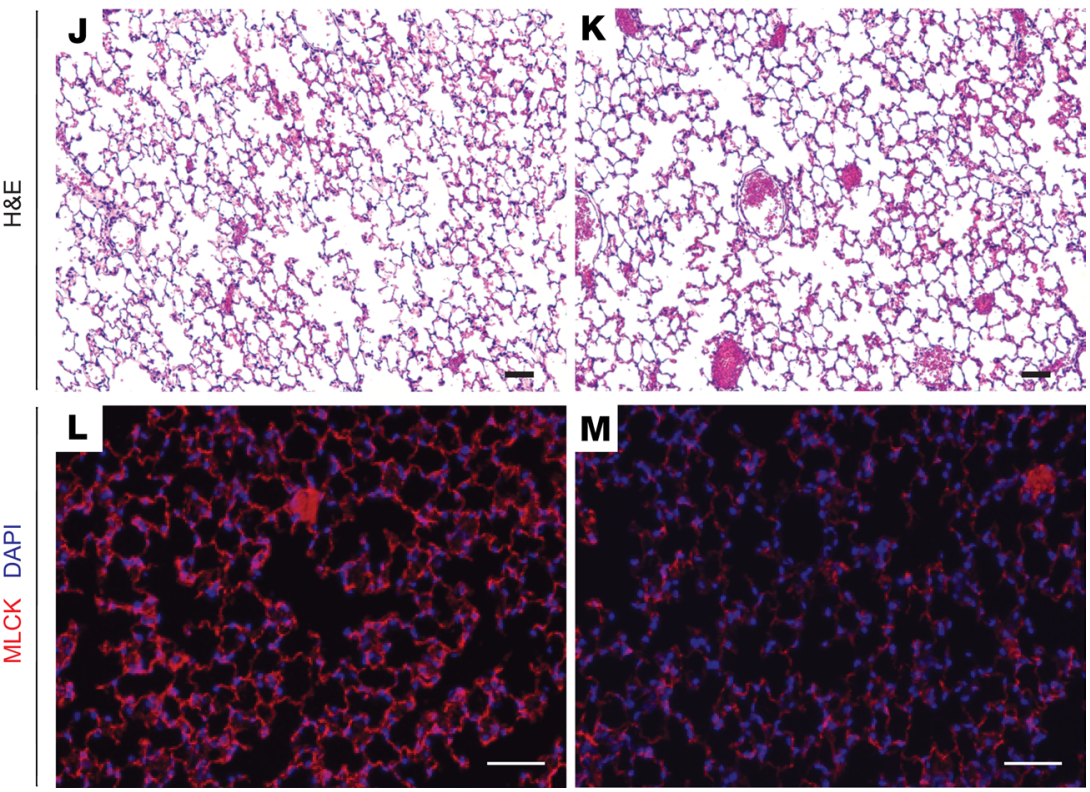

Figure 4. MIck requirement for septa formation is restricted to phase 1 but not phase 2 of alveologenesis. (A) Timeline of dox-induced gene inactivation and analysis in phase 1. All data and images are from P8 lungs. (B and C) Representative H\&E-stained sections. (D-G) Representative immunofluorescence staining for $\alpha$-SMA ( $\mathbf{D}$ and $\mathbf{E}$ ) or elastin ( $\mathbf{F}$ and $\mathbf{G}$ ) showing abnormal patterns in the mutant lungs. (H) Quantification of alveolar simplification by MLI. ${ }^{*} P=0.0223$ $(n=3)$. (I) Timeline of dox-induced gene inactivation and analysis in phase 2. All data are from P40 lungs. (J and $\mathbf{K})$ Representative H\&E-stained sections. ( $\mathbf{L}$ and $\mathbf{M})$ Representative immunofluorescence staining for MLCK (red) in the alveolar region showing efficient inactivation in mutant lungs. (N) Quantification of alveolar density by MLI. $P=0.24(n=3)$. Data represent the mean \pm SEM. $P$ values were determined by 2-tailed Student's $t$ test. Scale bars: $50 \mu$ m. 
and alveologenesis (21-23). We first tested whether TGF- $\beta$ pathway components were altered in Mlck-mutant lungs. Quantitative PCR (qPCR) analysis revealed that several of these genes were significantly downregulated in Mlck-mutant lungs (Supplemental Figure 2A). Furthermore, we observed a decrease in staining for p-SMAD3, a reporter of TGF- $\beta$ activity, in Mlck-mutant lung compared with control lungs (Supplemental Figure 2, B, C, E, and F). Western immunoblot analysis also showed that $\mathrm{p}-\mathrm{SMAD} 3$ protein levels were significantly reduced in $M l c k$-mutant lungs compared with levels in control lungs (Supplemental Figure 2, L and M). To test whether the decrease in TGF- $\beta$ signaling contributes to alveolar simplification in Mlck-mutant lungs in vivo, we induced overexpression of $\mathrm{Tg} f \beta 1$ in the mesenchyme by introducing a tet $\mathrm{O}-\mathrm{Tg}$ $f \beta 1$ allele into the Mlck mutant (24). We found an increase in p-SMAD3 staining, indicating an effective boost of TGF- $\beta$ activity (Supplemental Figure 2, D and G). Despite this, the alveolar simplification phenotype was not attenuated (Supplemental Figure 2, $\mathrm{H}-\mathrm{K})$. Although these results do not rule out the possibility that a decrease in TGF- $\beta$ signaling can contribute to the alveolar simplification phenotype in Mlck-mutant lungs, they do not indicate a significant role for this signaling.

Mlck-mutant lungs exhibited decreased YAP. The Hippo pathway plays a key role in mechanotransduction. YAP and transcriptional coactivator with PDZ-binding motif (TAZ) are central transcription effectors in Hippo signaling. Cell contractility and actin filament assembly stimulate activation and/or translocation of YAP and TAZ to the nucleus $(25,26)$. Previous studies have shown that in vitro inhibition of MLCK in cultured NIH3T3 and mesenchymal stem cells (MSCs) leads to reduced nuclear YAP $(27,28)$. To determine whether MLCK regulates YAP in alveolar myofibroblasts in vivo, we first performed immunostaining. We found that YAP staining was substantially diminished in Mlckmutant lungs (Figure 5, A and B). This was corroborated by Western immunoblot analysis, which showed that YAP protein levels were significantly reduced in Mlck-mutant lungs (Figure 5, C and D). Furthermore, qPCR analysis showed that Yap mRNA levels were also reduced in $M l c k$-mutant lungs (Figure 5E). To test whether YAP downregulation contributes to alveolar simplification, we first inactivated Yap and Taz in myofibroblasts using the Pdgfra ${ }^{\text {rtTA }}$ tetO-Cre combination driver (hereafter referred to as Yap Taz mutants) (7). Administration of dox from E9.5 to E18.5 did not lead to a change in lung size or histology (Supplemental Figure 3, A-D). However by P8, the Yap Taz mutant lungs had phenotypes similar to those of Mlck-mutant lungs, including simplified alveoli (Figure 5, F-H and M), abnormal $\alpha$-SMA patterns (Figure 5, $\mathrm{I}$ and J), and decreased p-MLC antibody staining (Figure 5, $\mathrm{K}$ and L). Western immunoblot analysis also showed that p-MLC protein levels were significantly reduced in Yap Taz-mutant lungs (Figure 5, N and O). Similar to Mlck, inactivation of Yap Taz in phase 1 of alveologenesis led to simplified alveoli (Supplemental Figure $3, \mathrm{E}-\mathrm{H})$. In a collagen contraction assay, myofibroblasts from Yap Taz-mutant lungs showed attenuated contraction compared with that seen in control cells (Supplemental Figure 3, I-M).

Overexpression of YAP attenuated alveolar simplification in Mlck-mutant lungs. To determine whether the decrease in YAP contributed to alveolar simplification in the Mlck-mutant lung in vivo, we overexpressed an activated form of YAP in the mesen- chyme of Mlck-mutant lungs by combining a tetO-Yap ${ }^{S 127 A}$-transgenic line with the Mlck mutant (29). Similar to what we observed in Tbx4-rtTA tetO-Cre Mlck ${ }^{f / f l}$-mutant lungs, inactivation of $M l c k$ specifically in myofibroblasts using Pdgfrat ${ }^{\text {rtTA }}$ tetO-Cre led to simplified alveoli (Figure 6, A and B), decreased YAP staining (Figure 6, D and E), abnormal $\alpha$-SMA patterns (Figure $6, \mathrm{G}$ and $\mathrm{H}$ ), and decreased p-MLC staining (Figure 6, J and K). Western immunoblot and qPCR analyses also confirmed that, like in Tbx4-rtTA tet $O$-Cre $M l c k^{f l / f l}$ mutant lungs, YAP protein and mRNA levels were significantly reduced in Pdgfra ${ }^{r t T A}$ tet $O$-Cre $M l c k^{f l / f l}$ lungs compared with controls (Supplemental Figure 4, A-C). Furthermore, overexpression of the activated form of YAP alone using Pdgfrat ${ }^{r t A}$ mice on a WT background did not alter alveologenesis (Supplemental Figure 5, A-C). We found that overexpression of YAP in the lungs of mice on the Pdgfra ${ }^{r t T A}$ tetO-Cre $M l c k^{f / f l}$-mutant background partially attenuated alveolar simplification, corrected $\alpha$-SMA patterns, and rescued p-MLC levels without affecting MLCK inactivation (Figure 6, C, F, I, and L-P). Together, these in vivo results suggest that the decrease in YAP expression was a significant contributor to impaired alveologenesis in $M l c k$-mutant lungs.

Inactivation of Mlck disrupted septa formation in PNX-induced lung regrowth. PNX, the surgical removal of 1 or more lung lobes, triggers compensatory regrowth in the remaining lobes, with increased cell proliferation and formation of new alveolar units. Elucidation of the molecular mechanisms has focused on signaling pathways that promote the proliferation and differentiation of AT2s after PNX (30-33). However, little is known about the mechanisms that regulate mesenchymal control of new septa formation after PNX. In a WT lung, consistent with prior findings (34), we induced $\alpha$-SMA in the alveolar mesenchyme of PNX lungs 6 days after resection (Supplemental Figure 6, A and B), indicating reactivation of myofibroblast properties.

Next, to determine whether MLCK-mediated myofibroblast contraction is required for PNX-induced reseptation, mice were injected with daily doses of dox at P40-P42 and were sacrificed on $\mathrm{P} 49$ for assessment of baseline phenotypes (Supplemental Figure $6 \mathrm{C}$ ). We found that following adult inactivation of $M l c k$, the mutant lungs were histologically indistinguishable from the control lungs (Supplemental Figure 6, D-F), despite efficient inactivation as demonstrated by MLCK antibody staining (Supplemental Figure 6, G and H).

We performed PNX 7 days after the last injection of dox, and the mice were sacrificed either 6 or 21 days after surgery (Figure 7A, Supplemental Figure 7A, and Supplemental Figure 8A). On day 6 after resection, compared with sham-operated control lungs, PNX-operated control lungs showed a slight but statistically significant increase in p-MLC staining intensity, whereas PNX-operated Mlck-mutant lungs had decreased p-MLC staining intensity, suggesting that alveolar stretch leads to MLCK-dependent increases in MLC phosphorylation (Supplemental Figure 7, A-E). Interestingly, compared with sham-operated control lungs, PNX-operated Mlck-mutant lungs exhibited similar AT2 cell proliferation rates and AT1/AT2 cell ratios compared with PNX-operated control lungs (Supplemental Figure 8, A-M). Consistent with these results, PNX-treated $M l c k$-mutant lungs underwent similar volume gains during compensatory lung regrowth (Figure 7, B and C). However, the expanded right lungs were exceedingly simpli- 

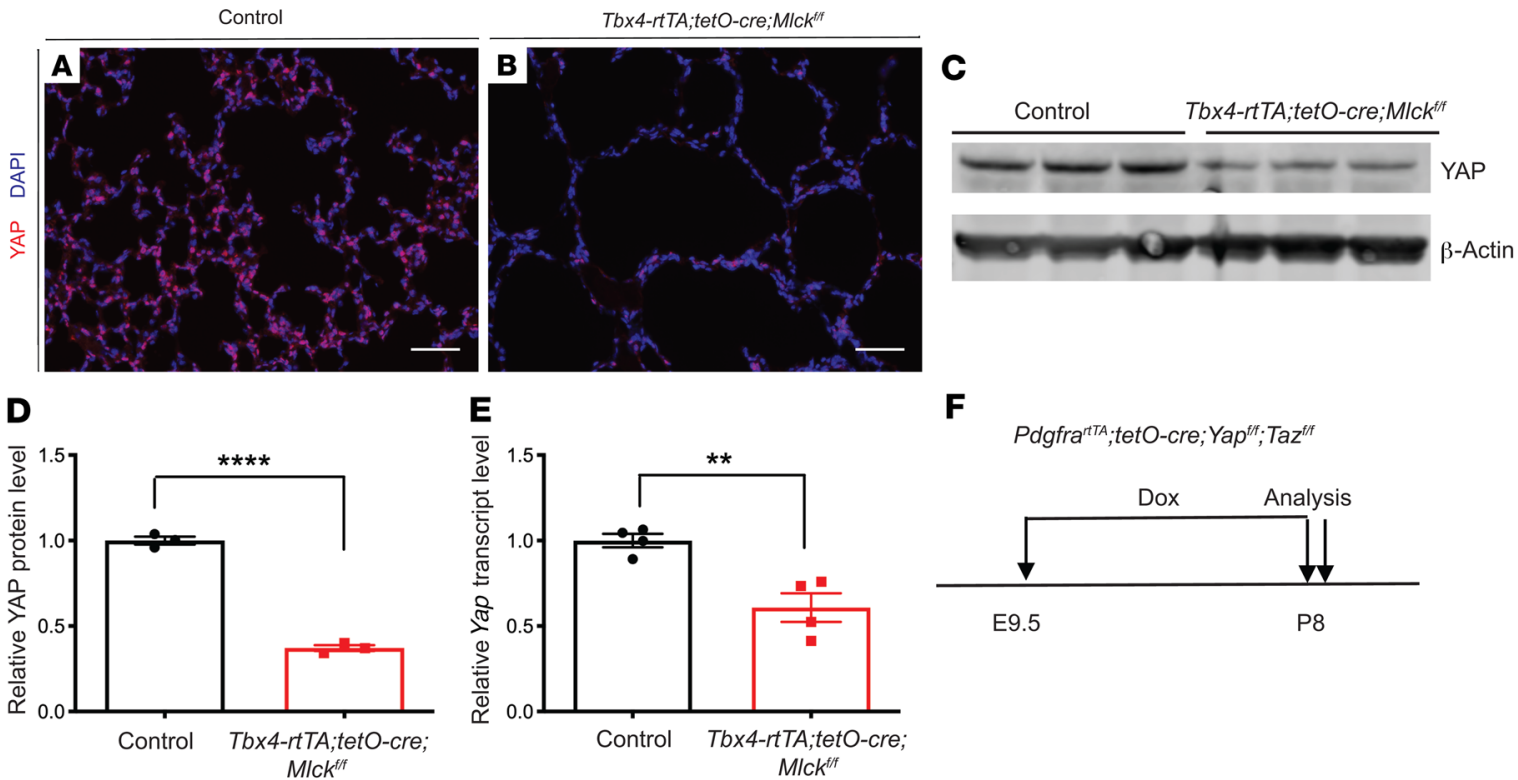

$\mathbf{F}$

Pdgfra ${ }^{\text {tTA }}$;tetO-cre; Yap ${ }^{f f ;} ; \mathrm{TaZ}^{f f f}$
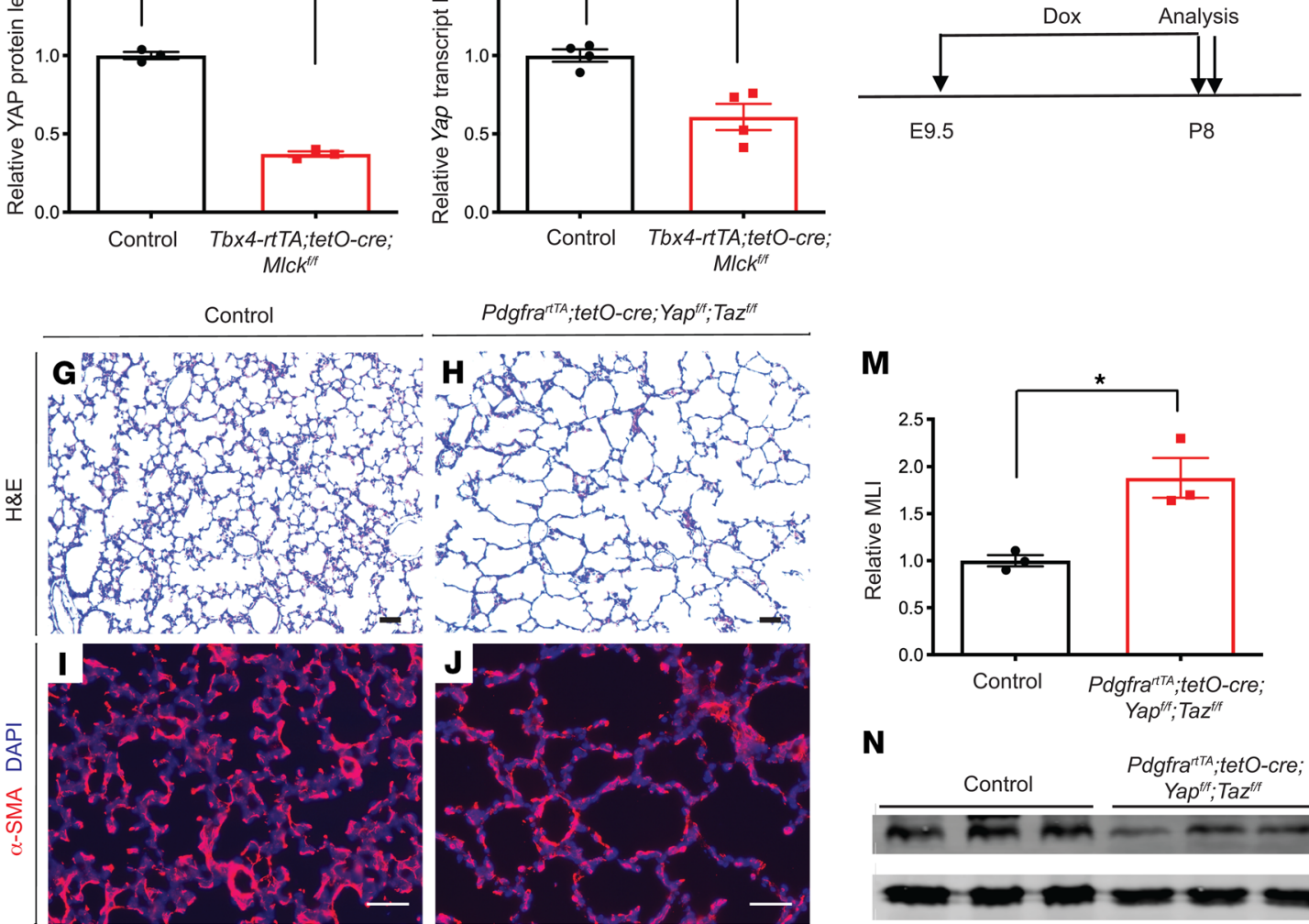

$\mathbf{M}$

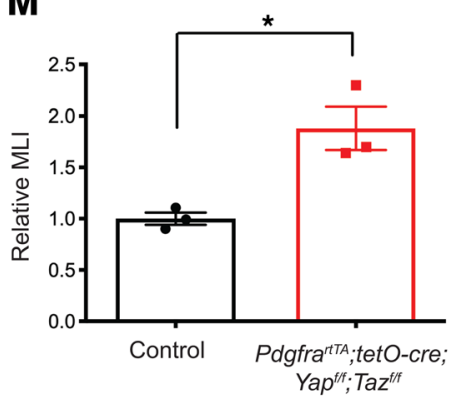

$\mathbf{N}$
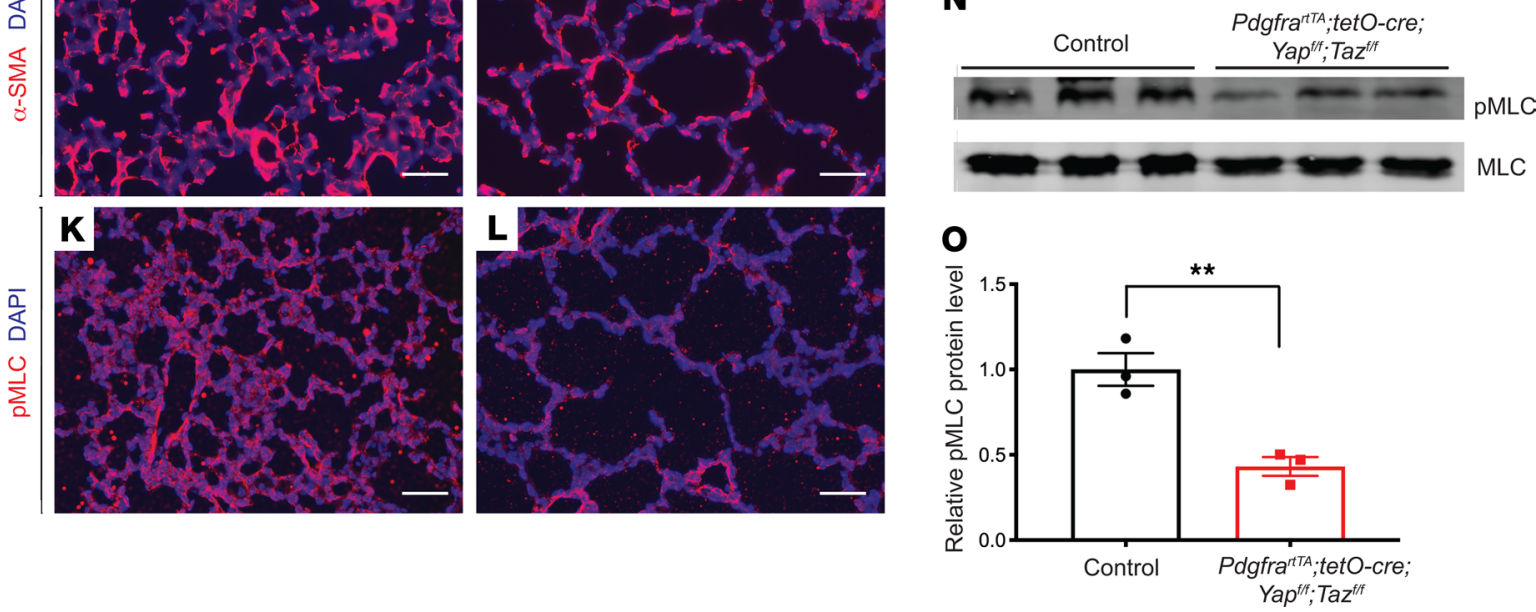

Figure 5. MIck-mutant lungs exhibit decreased YAP, and inactivation of Yap leads to alveolar simplification, similar to the Mlck mutant. (A and B) Representative immunofluorescence staining for total YAP (red) in the alveolar region showing mutant lungs with decreased YAP on P8. (C and D) Western blot analysis and quantification indicated that YAP levels were decreased in mutant lungs compared with controls on P8. ${ }^{* * *} P=0.000045$ $(n=3)$. $\beta$-Actin was used as a control. (E) qPCR showed that mutant lungs exhibited decreased Yap transcript levels on P8. ${ }^{* *} P=0.0053(n=4)$. $(\mathbf{F})$ Timeline of dox-induced inactivation and analysis. ( $\mathbf{G}$ and $\mathbf{H})$ Representative H\&E-stained sections from the alveolar regions of P8 lungs showed simplified alveoli in Yap Taz-mutant lungs. (I and J) Representative immunofluorescence staining for $\alpha$-SMA (red) in the alveolar region show that mutant lungs had abnormally localized myofibroblasts on P8. (K and $\mathbf{L}$ ) Representative immunofluorescence staining for $p-M L C$ (red) in the alveolar region show that mutant lungs had decreased MLC phosphorylation. (M) Quantification of alveolar simplification by MLI. ${ }^{*} P=0.0157(n=3)$. (N and 0) Western blot analysis and quantification indicated that the ratio of $p-M L C$ to MLC levels was decreased in the mutant lungs compared with control lungs on P8. ${ }^{* *} P=0.0067(n=3)$. Data represent the mean \pm SEM. $P$ values were determined by 2-tailed Student's $t$ test. Scale bars: $50 \mu \mathrm{m}$. 
fied in PNX-treated Mlck-mutant lungs compared with control lungs (Figure 7, D-J). These data demonstrate that Mlck is essential for reseptation following PNX.

\section{Discussion}

Alveologenesis is the final step of lung development and is responsible for the construction of approximately $95 \%$ of the gas-exchange surface area. This expansion is achieved by repeated formation of septal ridges that subdivide saccules into alveoli. In this study, we investigated the fundamental mechanism of septa formation. Our findings demonstrated that MLCK-mediated myofibroblast contraction was critical for septa formation not only in alveologenesis but also during alveolar regrowth following PNX. Furthermore, we found that YAP, the core transcriptional effector of the Hippo pathway, was regulated by MLCK during septa formation. YAP in turn worked together with MLCK to control MLC phosphorylation, myofibroblast contraction, and septa formation (Figure 7K).

Our model showing that contraction of the "fishnet" involves interconnected myofibroblasts is a departure from conventional models of septa formation. The previous models, based on 2D depictions of the septal crest as a finger-like protrusion, proposed that septa arise either through repulsion of cells into the alveolar lumen or through extension of the tip or base $(6,10-12)$. To our knowledge, no repulsion factor has been identified to date. Although retinoic acid and FGF signaling have been discussed as forming a signaling gradient in the extension models (sprouting at the tip or elongation at the base), it remains unclear how their primary role in elastogenesis translates into directing septal growth $(11,12,35)$. Regardless of the lack of clear molecular mechanisms to support these models, the "fishnet" pattern offers a more accurate illustration of the 3D alveolar architecture (13). This led us to hypothesize that coordinated contraction of the $\alpha$-SMA ${ }^{+}$myofibroblast "strings" of the net would either drive the ridges underlaid by the net into the lumen and/or provide tensile force to hold against air pressure that pushes on the alveolar surface. Through either or both modes, the alveolar surface underlaid by string-like myofibroblasts would form the alveolar entrance ring. Concomitantly, the surface area at the "pores" of the net would evaginate to form the wall and base of the alveoli. Our finding that MLCK-mediated myofibroblast contraction is essential for septa formation identified a core element that drives this morphogenesis event and, we believe, offers the first direct support for a model of alveologenesis.

Data from our group and others have also shown that $\alpha$-SMA staining signal was detectable in the alveolar mesenchyme in phase 1 (P3-P14) but not phase 2 (P15-P39) of alveologenesis (13). This restricted pattern of $\alpha$-SMA expression correlated with the requirement for $M l c k$ in phase 1 but not phase 2 of alveologenesis. These findings indicate fundamental mechanistic differences in how new septa arise in the 2 phases of alveologenesis. It remains unclear what drives septa formation in phase 2. Continued elaboration of the vasculature and/or fine-tuning of the elastin fiber network represent possible mechanisms.

Our finding that YAP/TAZ functions downstream of MLCK provides a link between Hippo pathway-mediated mechanotransduction to phosphorylation of MLC, myofibroblast contraction, and alveologenesis. Given that the alveoli are constantly under expansion/contraction with each inhalation/exhalation cycle, this link to mechanotransduction is significant. Previous studies have shown that Hippo pathway genes control lung morphogenesis in the epithelium (36-40) and pericytes (41). Here we show that inactivation of Yap Taz using Pdgfra ${ }^{\text {rtTA }}$ tetOCre led to simplified alveoli, demonstrating a requirement for Yap Taz in myofibroblasts. Epithelium-specific inactivation of Yap led to decreased p-MLC, presumably because of decreased expression of contractility factors such as Arhgef17 that resulted in disruption of branching morphogenesis (39). We show that myofibroblast-specific inactivation of Yap Taz led to decreased $\mathrm{p}-\mathrm{MLC}$ in the mesenchyme. YAP regulation of $\mathrm{p}-\mathrm{MLC}$ can occur in a MLCK-independent manner, as supported by restoration of p-MLC levels when activated Yap was expressed in Mlck-mutant lungs. We speculate that YAP may regulate p-MLC through regulation of enzymes such as Rho-associated protein kinase (ROCK) or MLCP (39). Overexpression of activated Yap in Mlck-mutant lungs also partially attenuated simplification, mechanistically connecting YAP and MLCK as 2 factors that act in parallel to promote MLC phosphorylation and septa formation.

PNX has been widely used in the lung to study the molecular mechanisms that control alveolar regeneration. Much of the attention has been focused on regrowth of the alveolar epithelium. Our data indicate that although myofibroblast contraction is not required for the gross volume expansion of the remaining lobes, it is essential for reseptation to "fill in" the expanded alveolar sacs. Stimulating new septa formation has remained the ultimate goal in the march to restore gas-exchange surface in diseases such as BPD and chronic obstructive pulmonary disease (COPD). Our findings indicate that, aside from retaining or regenerating myofibroblast progenitors, stimulating them to regain contractile property is critical. MLCK- and YAP-based promotion of MLC phosphorylation suggests an entry point to address this challenge.

\section{Methods}

Mice. Tbx4-rtTA (19), tetO-Cre (42), Mlck ${ }^{l l}$ (16), Yap ${ }^{f l}$ (43), Taz ${ }^{l l}$ (44), tetO-Yap ${ }^{\text {S127A }}$ (29), Pdgfra ${ }^{r T A}$ (7), Pdgfra ${ }^{G F P}$ (20), Rosa ${ }^{m T m G}$ (18), and tetO-Tgf $\beta 1$ (24) mice have all been previously described. Noon of the day on which a virginal plug was detected was considered to be E0.5. The day on which the mice were born was considered as PO. Prenatal reverse tet transcriptional activator (rtTA) activity was induced by dox administration starting at different gestational periods by feeding dox food to pregnant females $(625 \mathrm{mg} / \mathrm{kg}$; TestDiet). In postnatal pups and adult mice, rtTA activity was induced by intraperitoneal injection of dox (100 $\mathrm{mg} / \mathrm{kg}$ body weight). For low-dose sparse labeling, rtTA activity was induced by intraperitoneal injection of dox (10 mg/kg body weight). ML-7 (MilliporeSigma) was intraperitoneally injected daily from P1 to $\mathrm{P} 7 \mathrm{at}$ a dose of $2.5 \mathrm{mg} / \mathrm{kg}$.

Histology and immunofluorescence staining. For histological analysis, postnatal mouse lungs were gravity inflated and fixed overnight with $4 \%$ paraformaldehyde diluted in PBS, and samples were embedded in paraffin for sectioning. Sections were stained using a standard $\mathrm{H} \& \mathrm{E}$ protocol. Quantification of lung simplification was performed using the MLI method as previously described (35). At least 3 lungs with 3 evenly spaced sections from each lung were used to calculate the MLI for mutant or control samples at each stage. All experiments were repeated using multiple litters. 


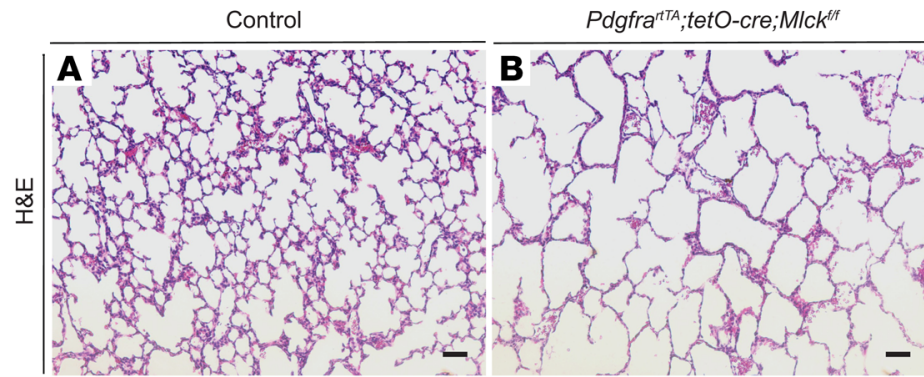

Pdgfrartia;tetO-cre;Mlck ;if;TetO-Yap ${ }^{\text {S127A }}$
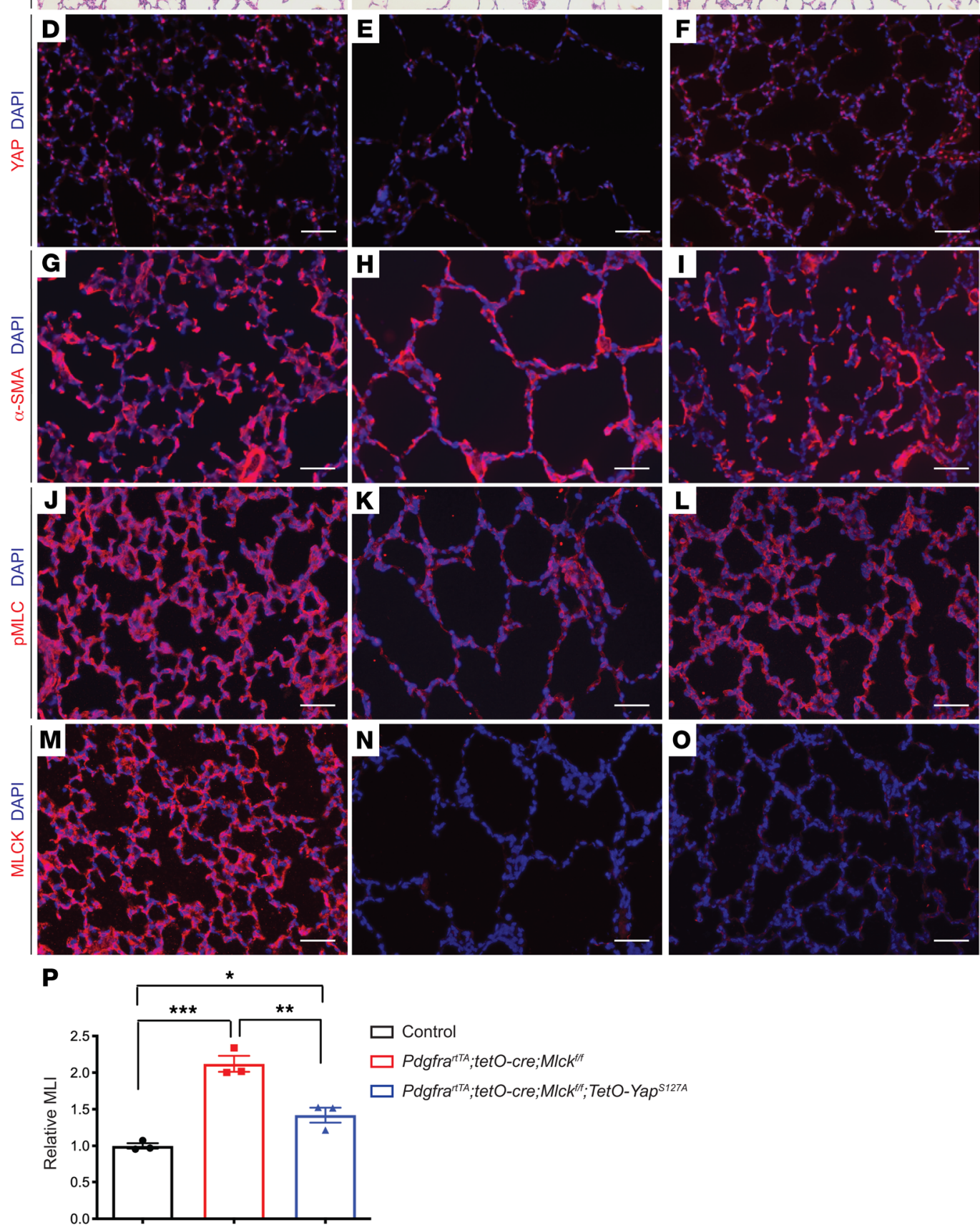

Figure 6. Overexpression of activated YAP using PdgfrartTA in Mlck-mutant lungs partially rescues phenotypes. (A-L) Representative images of stained sections from the alveolar regions of P8 lungs showing that Yap overexpression in Mlck-mutant lungs attenuated multiple phenotypes, including alveolar simplification (A-C), YAP staining intensity (D-F), $\alpha$-SMA patterns (G-I), and p-MLC staining intensity (J-L). (M-O) Representative immunofluorescence staining for MLCK (red) in the alveolar region of P8 lungs showed a similar extent of MLCK inactivation in the Mlck-mutant lungs with or without Yap over-

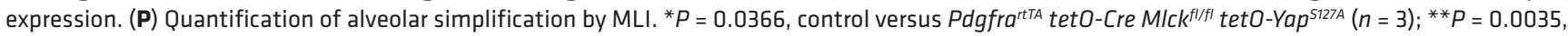

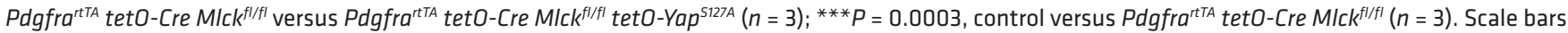
$50 \mu \mathrm{m}$. Data represent the mean \pm SEM. $P$ values were determined by 1-way ANOVA with Tukey's method to adjust for multiple comparisons. 
A

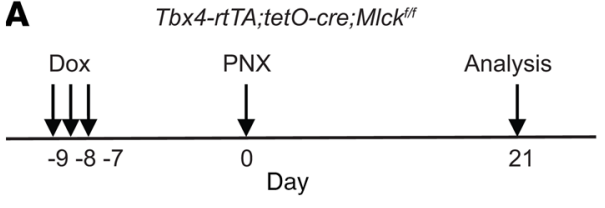

C

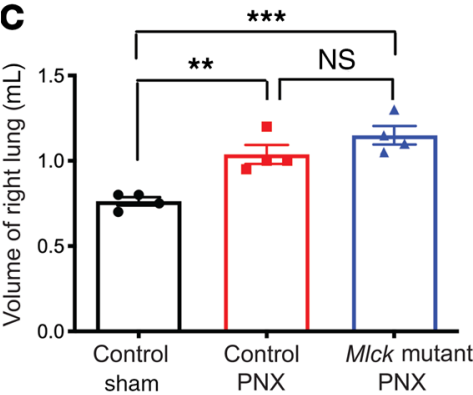

B

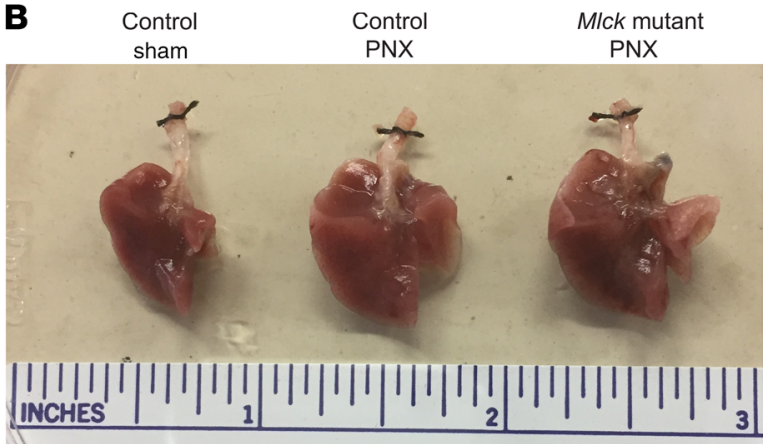

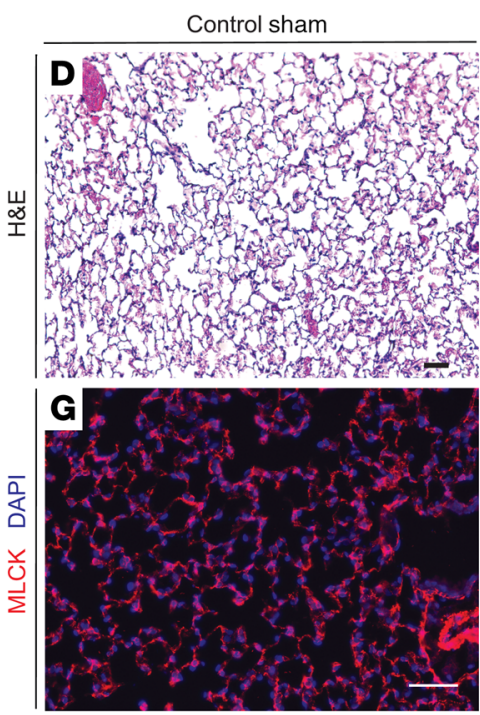

Control PNX
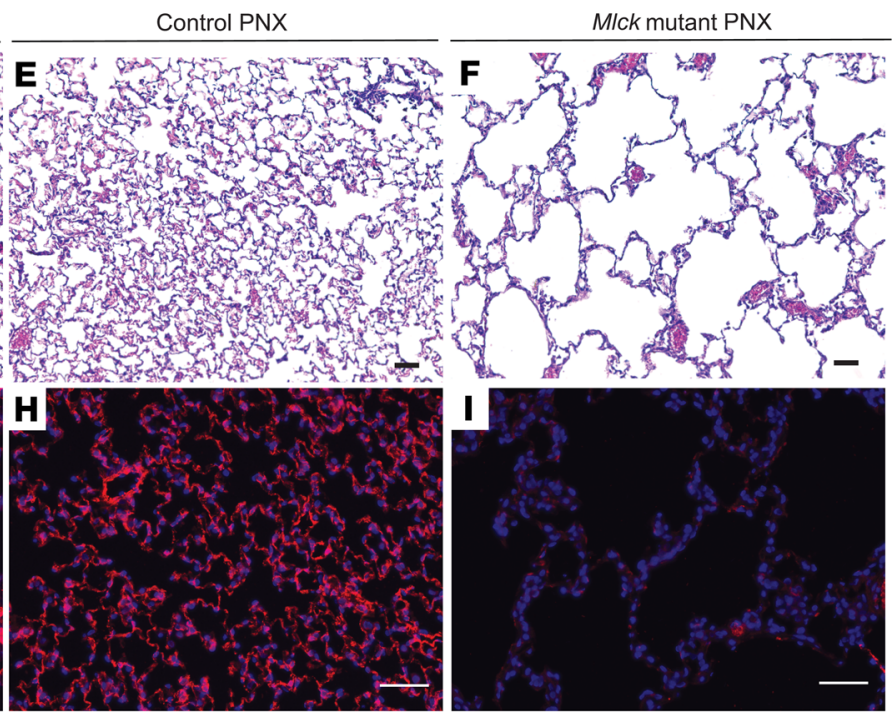

$\mathbf{J}$

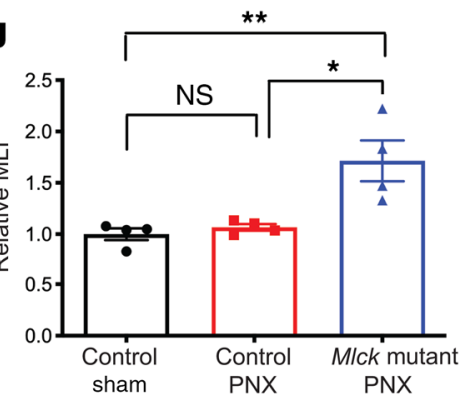

$\mathbf{K}$

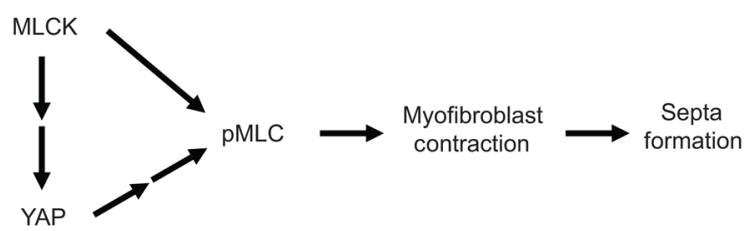

Figure 7. Inactivation of MIck disrupts reseptation during PNX-induced lung regrowth. (A) Timeline of dox-induced gene inactivation, PNX procedure, and analysis. (B) Whole-mount images of lungs on day 21 following PNX. (C) Quantification of the volume of the remaining right lung on day 21 following PNX. $P=0.2569$ (NS), control PNX versus Mlck-mutant PNX $(n=4) ;{ }^{* *} P=0.0062$, control sham versus control PNX ( $\left.n=4\right) ;{ }^{* *} P=0.0006$, control sham versus Mlck-mutant PNX $(n=4)$. (D-F) Representative H\&E-stained sections from alveolar regions showing extensive simplification in the mutant lung following PNX. (G-I) Representative immunofluorescence staining for MLCK (red) in the alveolar region, showing clear loss of MLCK in the mutant lung. (J) Quantification of alveolar simplification by MLI. $P=0.9186$ (NS), control sham versus control PNX $(n=4)$; ${ }^{*} P=0.0109$, control PNX versus $M I c k$-mutant PNX ( $\left.n=4\right)$; ${ }^{*} P=0.0061$, control sham versus Mlck-mutant PNX $(n=4)$. (K) During alveologenesis, MLCK functions in part through regulation of YAP and controls MLC phosphorylation, myofibroblast contraction, and septa formation. Data represent the mean \pm SEM. $P$ values were determined by 1 -way ANOVA with Tukey's method to adjust for multiple comparisons. Scale bars: $50 \mu \mathrm{m}$.

Immunofluorescence staining was performed following standard protocols, and stained sections were mounted using VECTASHIELD medium with DAPI (Vector Laboratories). For 3D immunofluorescence staining or detection of endogenous fluorescence signal, lungs were cut into 70 - $\mu$ m-thick sections, and $z$-stacks were collected using a Zeiss 880 Airyscan confocal microscope and then processed as 3D images using IMARIS analysis software (Bitplane, Switzerland) with no averaging. For E-cadherin whole-mount immu- 
nohistochemical staining, whole E13.5 embryos were fixed overnight in $4 \%$ paraformaldehyde diluted in PBS, and samples were stained as whole mounts using an ImmPACT SG Immunohistochemistry Kit (Vector Laboratories, SK-4705) following standard protocols. The following primary antibodies were used: MLCK (MilliporeSigma, M7905, 1:200), p-MLC (Abcam, ab2480, 1:200), $\alpha$-SMA (MilliporeSigma, F3777, 1:400), elastin (Abcam, ab21600, 1:500), YAP (Cell Signaling Technology, 4912, 1:100, recognizing both phosphorylated and nonphosphorylated forms), PDPN (Developmental Studies Hybridoma Bank, 8.1.1, 1:500), SPC (Seven Hills Bioreagents, WRAB-9337, 1:200), ICAM2 (BD, 553325, 1:200), PDGFR $\beta$ (Cell Signaling Technology, 3169, 1:200), IsoB4 (MilliporeSigma, L2895, 1:200 ), ADRP (Abcam, ab52356, 1:200), p-SMAD3 (MilliporeSigma, 07-1389, 1:200), Ki67 (Invitrogen, Thermo Fisher Scientific, 14-5698-82, 1:200), HOPX (Santa Cruz Biotechnology, sc-30216, 1:200), and E-cadherin (Cell Signaling Technology, 3195s, 1:100). The following secondary antibodies were used: goat anti-rabbit CY3 (Jackson ImmunoResearch, 111-165-144, 1:200), goat anti-mouse CY3 (Jackson ImmunoResearch, 115-165-003, 1:200), goat antirat CY3 (Jackson ImmunoResearch, 112-165-003, 1:200), and goat anti-Syrian hamster FITC (Jackson ImmunoResearch, 107-095-142, 1:200). A tyramide amplification system (Thermo Fisher Scientific, B40935) was used for the immunofluorescence staining experiment with the YAP antibody.

$q P C R$. Total RNA was extracted from lungs using TRIzol (Invitrogen, Thermo Fisher Scientific) and an RNeasy Mini RNA Extraction Kit (QIAGEN). RNA was reverse transcribed using the iScript Select cDNA Synthesis Kit (Bio-Rad). qPCR was performed using SYBR Green (Bio-Rad). Transcripts of target genes were normalized to $A c t b$ transcript levels. Data are shown as the average of a minimum of 3 biological replicas. The qPCR primers used are listed in Supplemental Table 1.

Western blot analysis. Lung tissues were homogenized in RIPA buffer supplemented with Complete Protease Inhibitor Cocktail tablets (Roche) and PhosSTOP Phosphatase Inhibitor Cocktail tablets (Roche). Protein samples were homogenized on a QIAGEN TissueLyser II and run on a $4 \%-12 \%$ SDS-PAGE gel, and then transferred onto PVDF membranes. The PVDF membranes were blocked in TBST with $5 \%$ dried milk powder or BSA (with $0.1 \%$ Tween-20) for at least 1 hour, incubated in the primary antibody overnight, and then incubated with the secondary antibody for 1 hour. Quantification was carried out using the Image Studio Lite software system (LI-COR Biosciences). The following primary antibodies were used: YAP (Cell Signaling Technology, 4912, 1:2000), $\beta$-actin (Novus Biologicals, NB600501, 1:5000), MLC (Cell Signaling Technology, 3672, 1:2000), p-MLC (Abcam, ab2480, 1:2000), SMAD2 and SMAD3 (Cell Signaling Technology, 8685, 1:2000), and p-SMAD3 (MilliporeSigma, 07-1389, 1:2000). The secondary antibodies used were: RDye 680RD donkey anti-mouse IgG (LI-COR, 925-69072, 1:10,000) and IRDye 800CW donkey anti-rabbit IgG (LI-COR, 925-32213, 1:10,000). Three biological replicates were performed for each antibody.

Collagen gel contraction assay. Pdgfra ${ }^{+}$myofibroblasts were sorted from Pdgfra ${ }^{G F P}$ mice on a control or Mlck-mutant background using flow cytometry as previously described (7). For Yap Tazmutant lungs, cells were stained using CD140a (PDGFR $\alpha$ ), phycoerythrin (PE) (eBioscience, 12-1401-81, 1:100). Briefly, minced lung was placed in $2 \mathrm{U} / \mathrm{mL}$ Dispase (STEMCELL Technologies) digestion solution and incubated in a $37^{\circ} \mathrm{C}$ shaker with frequent agitation for 30 minutes. Cell solution was filtered through a $40-\mathrm{mm}$ cell strainer (BD Falcon), and RBC lysis buffer (Abcam) was used to remove blood cells. Cell pellets were resuspended in FACS buffer, and cell sorting was performed on a FACSAria II (BD Biosciences). Sorted cells were collected in DMEM plus $10 \% \mathrm{FBS}$, and $5 \times 10^{4}$ cells were mixed with $62.5 \mu \mathrm{L}$ rat collagen 1 (Cultrex, $5 \mathrm{mg} / \mathrm{mL}$ ) and seeded onto a 48 -well plate. The following day, the gels were carefully transferred onto a 24-well plate with $500 \mu \mathrm{L}$ media per well and then imaged using a dissecting microscope with a camera every 24 hours for 3 days. Areas of collagen gel size were analyzed with Image (NIH).

$P N X$. Procedures were performed as previously described (45). Briefly, 8-week-old mice were anesthetized with ketamine (100 $\mathrm{mg} / \mathrm{kg})$ and xylazine $(10 \mathrm{mg} / \mathrm{kg})$ and then intubated and ventilated using a miniventilator (Kent Scientific). The chest wall was incised at the fourth intercostal space, and the left lung lobe was gently lifted from the chest, tightly ligated at the hilum with silk, and resected. The chest incision was then carefully sutured. Mice were disconnected from the ventilator when autonomous breathing recovered. Sham-operated animals underwent an identical procedure without removal of the left lobe.

Statistics. Comparisons between 2 groups were made using a 2-tailed, unpaired Student's $t$ test. Comparisons between 3 groups were made using a 1-way ANOVA with Tukey's method to adjust for multiple comparisons. Comparisons between 3 groups under 2 or more conditions were made using a 2-way ANOVA with Tukey's method or Sidak's method to adjust for multiple comparisons. All analyses were performed with GraphPad Prism 8 (GraphPad Software). Data are presented as the mean \pm SEM, and results were considered statistically significant if $P$ was less than 0.05 .

Study approval. This study was approved by the UCSD Animal Care and Use Committee and conformed to the Guide for the Care and Use of Laboratory Animals (National Academies Press, 2011). All animals were handled according to approved IACUC protocols (S16187) at UCSD. The mice were housed and all experimental procedures were performed in an American Association for Accreditation of Laboratory Animal Care-accredited laboratory animal facility at UCSD.

\section{Author contributions}

RL curated data, conducted studies, wrote the original draft of the manuscript, and helped review and edit the manuscript. XL, JH, and MSZ provided resources and helped review and edit the manuscript. XS curated data, supervised the study, acquired funding, and helped review and edit the manuscript.

\section{Acknowledgments}

We thank all the members of the Sun laboratory for insightful discussions, Pradipta Ghosh's laboratory for the use of the LI-COR imager, and the UCSD microscopy core for the use of the confocal microscope and IMARIS software. This work was supported by National Heart, Lung, and Blood Institute (NHLBI) grants R01 HL142215, HL143256, HL122406, and HL119946 and the grant for UCSD microscopy core (NINDS P30 NS047101).

Address correspondence to: Xin Sun, 9500 Gilman Drive, MC0766, CMME, Room 1055, San Diego, California 92093-0766, USA. Phone: 858.246.2000; Email: xinsun@ucsd.edu. 
1. Gehr P, Bachofen M, Weibel ER. The normal human lung: ultrastructure and morphometric estimation of diffusion capacity. Respir Physiol. 1978;32(2):121-140.

2. Davidson LM, Berkelhamer SK. Bronchopulmonary dysplasia: chronic lung disease of infancy and long-term pulmonary outcomes. J Clin Med. 2017;6(1):E4.

3. Mund SI, Stampanoni M, Schittny JC. Developmental alveolarization of the mouse lung. Dev Dyn. 2008;237(8):2108-2116.

4. Pozarska A, et al. Stereological monitoring of mouse lung alveolarization from the early postnatal period to adulthood. Am J Physiol Lung Cell Mol Physiol. 2017;312(6):L882-L895.

5. Boström H, et al. PDGF-A signaling is a critical event in lung alveolar myofibroblast development and alveogenesis. Cell. 1996;85(6):863-873.

6. Lindahl $\mathrm{P}$, et al. Alveogenesis failure in PDGF-A-deficient mice is coupled to lack of distal spreading of alveolar smooth muscle cell progenitors during lung development. Development. 1997;124(20):3943-3953.

7. Li R, Bernau K, Sandbo N, Gu J, Preissl S, Sun X. Pdgfra marks a cellular lineage with distinct contributions to myofibroblasts in lung maturation and injury response. Elife. 2018;7:e36865.

8. McGowan SE, McCoy DM. Regulation of fibroblast lipid storage and myofibroblast phenotypes during alveolar septation in mice. Am J Physiol Lung Cell Mol Physiol. 2014;307(8):L618-L631.

9. Andrae J, Betsholtz C, Gouveia L. PDGFR alpha signaling is required for alveolar development in the mouse lung. Mech Develop. 2017;145:S147.

10. Prodhan P, Kinane TB. Developmental paradigms in terminal lung development. Bioessays. 2002;24(11):1052-1059.

11. Pierce RA, Michael Shipley J. Retinoid-enhanced alveolization: identifying relevant downstream targets. Am J Respir Cell Mol Biol. 2000;23(2):137-141.

12. [No authors listed]. Functional genomics of lung disease. Second annual Pittsburgh International Lung Conference, October 2003. Am J Respir Cell Mol Biol. 2004;31(2 Suppl):S1-S81.

13. Branchfield K, Li R, Lungova V, Verheyden JM, McCulley D, Sun X. A three-dimensional study of alveologenesis in mouse lung. Dev Biol. 2016;409(2):429-441.

14. Emery JL. The post natal development of the human lung and its implications for lung pathology. Respiration. 1970;27:Suppl:41-Suppl:50.

15. Tomasek JJ, Gabbiani G, Hinz B, Chaponnier C, Brown RA. Myofibroblasts and mechano-regulation of connective tissue remodelling. Nat Rev Mol Cell Biol. 2002;3(5):349-363.

16. He WQ, et al. Myosin light chain kinase is central to smooth muscle contraction and required for gastrointestinal motility in mice. Gastroenterology. 2008;135(2):610-620.

17. Zhang WC, et al. Myosin light chain kinase is necessary for tonic airway smooth muscle contraction. J Biol Chem. 2010;285(8):5522-5531.

18. Muzumdar MD, Tasic B, Miyamichi K, Li L, Luo L. A global double-fluorescent Cre reporter mouse. Genesis. 2007;45(9):593-605.

19. Zhang W, et al. Spatial-temporal targeting of lung-specific mesenchyme by a Tbx4 enhancer. BMC Biol. 2013;11:111.

20. Hamilton TG, Klinghoffer RA, Corrin PD, Soriano P. Evolutionary divergence of platelet-derived growth factor alpha receptor signaling mechanisms. Mol Cell Biol. 2003;23(11):4013-4025.

21. Wipff PJ, Rifkin DB, Meister JJ, Hinz B. Myofibroblast contraction activates latent TGF-beta1 from the extracellular matrix. JCell Biol. 2007;179(6):1311-1323.

22. Chen $\mathrm{H}$, et al. Abnormal mouse lung alveolarization caused by Smad3 deficiency is a developmental antecedent of centrilobular emphysema. Am J Physiol Lung Cell Mol Physiol. 2005;288(4):L683-L691.

23. Xu B, et al. Molecular mechanisms of MMP9 overexpression and its role in emphysema pathogenesis of Smad3-deficient mice. Am J Physiol Lung Cell Mol Physiol. 2012;303(2):L89-L96.

24. Liu X, Alexander V, Vijayachandra K, Bhogte E, Diamond I, Glick A. Conditional epidermal expression of TGFbeta 1 blocks neonatal lethality but causes a reversible hyperplasia and alopecia. Proc Natl Acad Sci USA. 2001;98(16):9139-9144.

25. Dupont S, et al. Role of YAP/TAZ in mechanotransduction. Nature. 2011;474(7350):179-183.

26. Totaro A, Panciera T, Piccolo S. YAP/TAZ upstream signals and downstream responses. Nat Cell Biol. 2018;20(8):888-899.

27. Wada K, Itoga K, Okano T, Yonemura S, Sasaki H. Hippo pathway regulation by cell morphology and stress fibers. Development. 2011;138(18):3907-3914.

28. Driscoll TP, Cosgrove BD, Heo SJ, Shurden ZE, Mauck RL. Cytoskeletal to nuclear strain transfer regulates YAP signaling in mesenchymal stem cells. Biophys J. 2015;108(12):2783-2793.

29. Camargo FD, et al. YAP1 increases organ size and expands undifferentiated progenitor cells. Curr Biol. 2007;17(23):2054-2060.

30. Ding BS, et al. Endothelial-derived angiocrine signals induce and sustain regenerative lung alveolarization. Cell. 2011;147(3):539-553.

31. Liu Z, et al. MAPK-Mediated YAP activation controls mechanical-tension-induced pulmonary alveolar regeneration. Cell Rep. 2016;16(7):1810-1819.
32. Lechner AJ, et al. Recruited monocytes and type 2 immunity promote lung regeneration following pneumonectomy. Cell Stem Cell. 2017;21(1):120-134.e7.

33. Chung MI, Bujnis M, Barkauskas CE, Kobayashi Y, Hogan BLM. Niche-mediated BMP/SMAD signaling regulates lung alveolar stem cell proliferation and differentiation. Development. 2018;145(9):dev163014.

34. Chen L, Acciani T, Le Cras T, Lutzko C, Perl AK. Dynamic regulation of platelet-derived growth factor receptor $\alpha$ expression in alveolar fibroblasts during realveolarization. Am J Respir Cell Mol Biol. 2012;47(4):517-527.

35. Li R, Herriges JC, Chen L, Mecham RP, Sun X FGF receptors control alveolar elastogenesis. Development. 2017;144(24):4563-4572.

36. Mahoney JE, Mori M, Szymaniak AD, Varelas $\mathrm{X}$, Cardoso WV. The hippo pathway effector Yap controls patterning and differentiation of airway epithelial progenitors. Dev Cell. 2014;30(2):137-150

37. Zhao R, et al. Yap tunes airway epithelial size and architecture by regulating the identity, maintenance, and self-renewal of stem cells. Dev Cell. 2014;30(2):151-165

38. Lange AW, Sridharan A, Xu Y, Stripp BR, Perl AK, Whitsett JA. Hippo/Yap signaling controls epithelial progenitor cell proliferation and differentiation in the embryonic and adult lung. $J \mathrm{Mol}$ Cell Biol. 2015;7(1):35-47.

39. Lin C, et al. YAP is essential for mechanical force production and epithelial cell proliferation during lung branching morphogenesis. Elife. 2017;6:e21130.

40. Nantie LB, et al. Lats1/2 inactivation reveals Hippo function in alveolar type I cell differentiation during lung transition to air breathing. Development. 2018;145(21):dev163105.

41. Kato K, et al. Pulmonary pericytes regulate lung morphogenesis. Nat Commun. 2018;9(1):2448.

42. Perl AK, Wert SE, Nagy A, Lobe CG, Whitsett JA. Early restriction of peripheral and proximal cell lineages during formation of the lung. Proc Natl Acad Sci USA. 2002;99(16):10482-10487.

43. Xin M, et al. Regulation of insulin-like growth factor signaling by Yap governs cardiomyocyte proliferation and embryonic heart size. Sci Signal. 2011;4(196):ra70.

44. Xin M, et al. Hippo pathway effector Yap promotes cardiac regeneration. Proc Natl Acad Sci USA. 2013;110(34):13839-13844

45. Liu Z, Fu S, Tang N. A standardized method for measuring internal lung surface area via mouse pneumonectomy and prosthesis implantation. J Vis Exp. 2017;(125):56114. 